\title{
ABSTRACT DIFFERENTIAL ALGEBRA AND THE ANALYTIC CASE. II
}

\section{A. SEIDENBERG}

Let $R$ be the rational number-field, $K=R\left\langle u_{1}, \cdots, u_{m}\right\rangle$ a finite ordinary differential extension-field of $R$. Let $u_{i j} \mapsto c_{i j}$ under an arbitrary abstract-field isomorphism $\sigma$ of $K$ onto a field $K_{1}$. Let the field of formal power-series $K_{1}((z))$ be converted into a differential field by placing $\left(\Sigma a_{j} z^{j} / j !\right)^{\prime}=\Sigma a_{j} z^{j-1} /(j-1)$ !. Then the assignment $u_{i} \mapsto \bar{u}_{i}$ $=\Sigma c_{i j} z^{j} / j$ ! defines a differential-field isomorphism of $K$ onto $R\left\langle\bar{u}_{1}, \cdots, \bar{u}_{m}\right\rangle$.

This is easily proved (see $[1$, p. 160]). We may note that on $R\left\{u_{1}, \cdots, u_{m}\right\}$ the mapping $\sigma$ is given by $\left.H(u) \mapsto H(\bar{u})\right|_{z=0}$ (for $H \in R\{U\})$. Thus if $\left.H(u) \mapsto H(\bar{u})\right|_{z=0}$ is an isomorphism, then $H(u)$ $\mapsto H(\bar{u})$ is an isomorphism. The converse is not true: thus suppose $m=1$ and $u=u_{1}$ is transcendental over $R$; then $H(u) \mapsto H(\bar{u}-\bar{u}(0))$ still yields an isomorphism, but $\left.H(u) \mapsto H(\bar{u}-\bar{u}(0))\right|_{z=0}$ does not.

As, however, in the proof of a corollary in $[1$, p. 162] we tacitly made use of this converse, a correction is necessary. This corollary reads:

Let $K, K_{1}$ be finite extensions of the rational number-field $R, K \subseteq K_{1}$, and let $\tau: K \rightarrow K^{*}$ be an isomorphism of $K$ with a field of meromorphic functions $K^{*}$. Then $\tau$ can be extended to an isomorphism $\tau: K_{1} \rightarrow K_{1}^{*}$ of $K_{1}$ with a field of meromorphic functions $K_{1}^{*}$.

In the definition of a field of meromorphic functions $K^{*}$, an open, connected region $A$ intervenes. If $A_{1}$ is an open, connected subregion of $A$, the mapping $\left.f \mapsto f\right|_{A_{1}}$ (for $f \in K^{*}$ ) yields an isomorphism of $K^{*}$. It is understood in the above corollary that $K^{*}$ may be replaced by any isomorphic copy obtained in this way.

Let $K=R\left\langle u_{1}, \cdots, u_{m}\right\rangle, \tau: u_{i} \mapsto \bar{u}_{i}$, and let $A$ be the region associated with $K^{*}=R\left\langle\bar{u}_{1}, \cdots, \bar{u}_{m}\right\rangle$. The functions $\bar{u}_{1}, \cdots, \bar{u}_{m}$ need not be holomorphic, but writing them as quotients of holomorphic functions, letting $P$ be a point of $A$ where the denominators do not vanish, and suitably restricting $A$ about $P$, we may assume that they are holomorphic. We may also assume that $P$ is given by $z=0$. Now consider the mapping $\left.H(u) \mapsto H(\bar{u})\right|_{z=0}$. This is always a homomorphism, but may fail to be an isomorphism. However, if $H(\bar{u}) \neq 0$, then $H(\bar{u})$ has only a finite number of zeros in a small circle about $P$; we propose to select a new $P$, avoiding these zeros. Since there are

Received by the editors March 19, 1969. 
only an enumerable number of $H$ in $R\{U\}$, we can choose a new $P$ so that $H(u) \neq 0$ implies $\left.H(\bar{u})\right|_{P} \neq 0$. Thus, although the converse spoken about is not true, we operate very much as though it were.

Let $K_{1}=K\left\langle v_{1}, \cdots, v_{n}\right\rangle$. Clearly we need consider only the case $n=1, K_{1}=K\langle v\rangle$. In case $v$ is transcendental over $K_{1}$, we take $\bar{v}$ $=\Sigma d_{j} z^{i} / j$ ! with $d_{0}, d_{1}, \cdots$, algebraically independent over $R\left(\cdots, c_{i j}, \cdots\right)$ and $\Sigma d_{j} z^{i} / j$ ! convergent, where $\bar{u}_{i}=\Sigma c_{i j} z^{i} / j$ ! and $\left.H(u) \mapsto H(\bar{u})\right|_{z=0}$ is an isomorphism after the preparations of the last paragraph. Then

$$
R\left(\cdots, c_{i j}, \cdots, d_{0}, d_{1}, \cdots\right) \simeq R\left(\cdots, u_{i j}, \cdots, v_{0}, v_{1}, \cdots\right)
$$

and $u_{i} \mapsto \bar{u}_{i}, v \mapsto \bar{v}$ defines an isomorphism of $K\langle v\rangle$ onto a field of meromorphic functions; this isomorphism extends $\tau$.

Now consider the case that $v$ is algebraic over $K$. By the previous case, we may assume that some $u_{i}$ are transcendental over $R$, by adjoining an extra $u$ if necessary, hence, by the theorem of the primitive element, may assume that $u_{1}, \cdots, u_{m-1}$ are algebraically independent and that $u_{m}$ and $v$ are primitive elements of $K$ and $K_{1}$ respectively over $R\left\langle u_{1}, \cdots, u_{m-1}\right\rangle$. After any such preparation, if we lose the assumption that $\left.H(u) \mapsto H(\bar{u})\right|_{z=0}$ is an isomorphism, we can always regain it. Now let $v, v_{1}, \cdots, v_{r-1}$ be algebraically independent (in the algebraic sense) over $R\left\langle u_{1}, \cdots, u_{m-1}\right\rangle$ and $v_{r}$ algebraic over $R\left\langle u_{1}, \cdots, u_{m-1}\right\rangle\left(v, \cdots, v_{r-1}\right)$. Let $\bar{v}=\Sigma d_{j} z^{j} / j$ ! be constructed so that $\bar{v}$ is convergent and $R\left(\left\{c_{i j}\right\}, \cdots,\left\{c_{m-1 j}\right\},\left\{d_{j}\right\}\right)$ $\simeq R\left(\left\{u_{i j}\right\}, \cdots,\left\{u_{m-1 j}\right\},\left\{v_{j}\right\}\right)$-see the proof of the Embedding Theorem [1, p. 160]. As in that proof, $\bar{v}$ is the unique solution of a differential equation $V_{r}-d_{r}=T\left(z ; V_{0}-d_{0}, \cdots, V_{r-1}-d_{r-1}\right)$ with specified initial conditions. Hence in this construction we can always replace $\left\{d_{j}\right\}$ by a conjugate $\left\{d_{j}^{*}\right\} / R\left(\cdots, c_{i j}, \cdots\right)$ $(i=1, \cdots, m-1)$ to get an equally good $\Sigma d_{j}^{*} z^{j} / j$ ! (i.e., $\Sigma d_{j}^{*} z^{j} / j$ ! will also be convergent, as it is the unique solution of the differential equation $V_{r}-d_{r}^{*}=T\left(z ; V_{0}-d_{0}^{*}, \cdots, V_{r-1}-d_{r-1}^{*}\right)$ with specified initial conditions).

Now we have an isomorphic mapping of $R\left\langle u_{1}, \cdots, u_{m-1}, v\right\rangle$ onto a field of meromorphic functions $R\left\langle\bar{u}_{1}, \cdots, \bar{u}_{m-1}, \bar{v}\right\rangle$; and contracting $A$, we may suppose $R\left\{\bar{u}_{1}, \cdots, \bar{u}_{m-1}, \bar{v}\right\}$ consists of holomorphic functions in $A$. This isomorphism agrees with $\tau$ on $u_{1}, \cdots, u_{m-1}$. However, it may send $u_{m}$ into a $u_{m}^{*} \neq \bar{u}_{m}$; in fact, $u_{m}^{*}$ may not even be holomorphic. (The possibility that $u_{m}^{*} \neq \bar{u}_{m}$ was not taken into account in $[1$, p. 162].) Changing the center $z=0$, we may assume $u_{m}^{*}$ is holomorphic, too; $u_{m}^{*}=\Sigma c_{m j}^{*} z^{j} / j$ !. Moreover we may assume 
$R\left(\left\{u_{1 j}\right\}, \cdots,\left\{u_{m-1 j}\right\},\left\{u_{m j}\right\},\left\{v_{j}\right\}\right)$

Now

$$
\simeq R\left(\left\{c_{1 j}\right\}, \cdots,\left\{c_{m-1 j}\right\},\left\{c_{m j}^{*}\right\},\left\{d_{j}\right\}\right) .
$$

$$
R\left(\left\{c_{1 j}\right\}, \cdots,\left\{c_{m-1 j}\right\},\left\{c_{m j}^{*}\right\}\right) \simeq R\left(\left\{c_{1 j}\right\}, \cdots,\left\{c_{m-1 j}\right\},\left\{c_{m j}\right\}\right)
$$

and this isomorphism can be extended to

$$
\begin{aligned}
R\left(\left\{c_{1 j}\right\}, \cdots,\left\{c_{m-1 j}\right\},\left\{c_{m j}^{*}\right\},\left\{d_{j}\right\}\right) & \\
& \simeq R\left(\left\{c_{1 j}\right\}, \cdots,\left\{c_{m-1 j}\right\},\left\{c_{m j}\right\},\left\{d_{j}^{*}\right\}\right) .
\end{aligned}
$$

These $d_{j}^{*}$ define a $\bar{v}^{*}=\Sigma d_{j}^{*} z^{j} / j$ !, which can replace $\bar{v}$. I.e., we have an isomorphism $R\left\langle u_{1}, \cdots, u_{m}, v\right\rangle \simeq R\left\langle\bar{u}_{1}, \cdots, \bar{u}_{m}, \bar{v}^{*}\right\rangle$. This isomorphism extends $\tau$ and the proof is complete.

Above we were assuming that $K, K_{1}$ were ordinary differential fields. However, similar considerations hold for partial differential fields.

\section{REFERENCE}

1. A. Seidenberg, Abstract differential algebra and the analytic case, Proc. Amer. Math. Soc. 9 (1958), 159-164.

University of California, Berkeley 